\title{
REVISTA BRASILEIRA DE ENERGIAS RENOVÁVEIS

\author{
ANÁLISE DO REGIME EÓLICO VISANDO A GERAÇÃO DE ENERGIA PARA \\ FINS AGRÍCOLAS NA CIDADE DE IPORÁ, GOIÁS, BRASIL ${ }^{1}$
}

\section{UÉLITON SOUSA FERREIRA ${ }^{2}$, VANESSA DE FÁTIMA GRAH PONCIANO ${ }^{3} \&$ ISAAC $^{2}$ DE MATOS PONCIANO ${ }^{4}$}

\begin{abstract}
${ }^{1}$ Publicado no Ano de 2019;
${ }^{2}$ Discente do Curso Bacharelado em Agronomia no Instituto Federal de Educação, Ciência e Tecnologias Goiano, Campus Iporá, uelitonsousa.95@ hotmail.com;

${ }^{3}$ Doscente do Instituto Federal de Educação, Ciência e Tecnologias Goiano, Campus Iporá;

${ }^{4}$ Doscente da Universidade de Rio Verde.
\end{abstract}

Resumo: Com o crescimento na demanda por energia elétrica, a geração eólica vem ganhando espaço na matriz energética do Brasil, por ser uma energia limpa e de fonte inesgotável, fonte esta, em que o Brasil apresenta alto potencial para a produção de energia em diversas regiões do país. Os dados de velocidade do vento foram obtidos por meio do Posto Meteorológico do INMET (Instituto Nacional de Meteorologia), instalado na Fazenda Escola do Instituto Federal Goiano, Iporá, Goiás, Brasil. A velocidade do vento foi medida com um anemômetro, instalado a dez metros de altura do solo, com registros entre os anos de 2014/2015. A variabilidade da velocidade do vento foi representada pela distribuição de frequência de Weibull, uma função de densidade de probabilidade de dois parâmetros (k e c). Foram utilizados os parâmetros k e c para correlacionar a função Gama com a velocidade do vento média anual, com a variância e com a densidade de potência média. Foi realizado um perfil de velocidade do vento para avaliar o comportamento da velocidade média histórica a partir da altura medida, para estimar o ganho de densidade de potência. Os valores de k, para todas as alturas, foram próximos de 1 o que corresponde a um regime de vento altamente variável, e os valores de c também foram baixos, representando uma baixa velocidade medida vento para o local. O local foi caracterizado como não sendo favorável para a aplicação de turbinas eólicas de pequena escala para geração de energia.

Palavras-chave: Cerrado, Energia eólica, Energias renováveis. 


\title{
ANALYSIS OF WIND REGIME AIMED AT GENERATING ENERGY FOR AGRICULTURAL PURPOSES IN THE CITY OF IPORÁ, GOIÁS, BRAZIL
}

\begin{abstract}
With the growth in demand for electricity, wind generation has been increasing in the energy matrix of Brazil, as a clean and inexhaustible energy source, in Brazil this source has a high potential for energy production in various regions of the country. The wind speed data were collected at the Meteorological Station INMET (Meteorological National Institute), the station was installed at Farm School at the Federal Institute Goiano, Iporá, Goiás, Brazil. The wind speed was measured by an anemometer installed at the height $10 \mathrm{~m}$ from the ground, with records between the years 2014/2015.The wind speed variability was represented by the Weibull frequency distribution, a probability density function of two parameters ( $\mathrm{k} \mathrm{e} \mathrm{c}$ ). We used the parameters $\mathrm{k}$ e c, to correlate the Gamma function with the annual average wind speed, the variance and power mean density. We made a wind profile to evaluate the behavior of historical average speeds at higher altitudes which the measured by anemometer, to estimate the gain in power density. The values of $\mathrm{k}$ for all heights were close to 1 which corresponds to a wind regime highly variable, and c values were also low representing a low average speed of the local. The location was characterized as being unfavorable for the application of small scale wind turbines for power generation.
\end{abstract}

Key words: Cerrado, Wind energy, Renewable energies.

\section{Introdução}

O crescimento na demanda por energia elétrica, associado ao fato de que a maior parte da energia produzida no mundo vem de fontes finitas, tem subsidiado pesquisas em todo mundo direcionadas a produção de energia limpa e de fontes inesgotáveis. Dentre os destaques de produção de energia renovável está a eólica, fonte esta, em que o Brasil apresenta alto potencial. De acordo com dados disponibilizados pela Agência Nacional de Energia Elétrica (ANEEL, 2016), o Brasil possui 9,315GW de capacidade instalada de energia eólica, o que corresponde à 6,29\% da matriz energética Brasileira. Dentre tantas vantagens, os investimentos com energia eólica têm gerado inúmeros benefícios financeiros para a economia Brasileira, entre eles, novos postos de trabalho, desenvolvimento social e econômico de regiões subdesenvolvidas e movimentação da indústria (BRASIL, 2016). 
Além dos benefícios econômicos, a energia eólica também contribui para a preservação do meio ambiente, com geração de energia sem emissão de dióxido de carbono, principal responsável pelo agravamento do efeito estufa e mudanças climáticas (ERTÜTK, 2012). No entanto, na luta contra o complexo desafio de cumprir os objetivos climáticos é necessário levar em consideração a contribuição de várias tecnologias de energias renováveis, em especial unidades de geração em pequena escala (GRIESER et al., 2015). Nesse contexto, de turbinas eólicas de pequena escala (TEE), os habitantes das comunidades rurais podem ser promissores usuários dessa tecnologia, principalmente aqueles que dependem da agricultura irrigada, pois, a maioria dos sistemas de irrigação demanda o fornecimento de energia elétrica para o seu funcionamento. Neste sentido, devido ao seu vasto potencial, a produção eólica tem sido um meio promissor para levar energia descentralizada onde as redes convencionais de energia não conseguem e também no intuito de reduzir a dependência do agricultor de fontes externas e caras de energia; proporcionando assim o desenvolvimento das áreas rurais. Segundo Albiero et al. (2014), uma forma de melhorar a renda da agricultura familiar é possibilitar o acesso à tecnologia de aero geradores que devem ser apropriados para cada cenário.

Devido aos recentes avanços da agricultura, a produção de energia deve acompanhar a crescente demanda no meio rural. Nesse contexto, a energia eólica se torna uma opção para a geração de energia, podendo ser uma saída para os avanços rurais das áreas carentes em aplicação de tecnologia, como o oeste do estado de Goiás(IICA, 2014). Diante desta realidade tecnológica, destaca-se a necessidade de estudos de potencial eólico, que possam auxiliar no avanço da produção de energia eólica para a região oeste de Goiás. Alguns estudos de potencial eólico estão disponíveis como, por exemplo, o Atlas de Potencial Eólico desenvolvido pelo Ministério de Minas e Energias em conjunto com outras instituições (AMARANTE et al., 2001), contudo este atlas foi desenvolvido com dados de velocidade do vento acima de $50 \mathrm{~m}$, o que não é interessante para a aplicação de TEEs. Por isso, faz-se necessário um estudo com velocidades medidas em alturas menos elevadas. Segundo Grieseret al. (2015) as TEEs são caracterizadas por baixa capacidade instalada (em torno de 1 a $10 \mathrm{~kW}$ ), altura do eixo da turbina aproximadamente a $30 \mathrm{~m}$ do nível do solo e podem estar instaladas isoladamente. Especificamente, as contribuições deste estudo são as seguintes:

(i) Análise estatística de uma série temporal de dados de velocidade horária do vento, coletada de uma estão meteorológica, por meio da função de distribuição de Weibull. 
(ii) A partir dos resultados estatísticos exibir um diagnóstico do potencial eólico para fins agrícolas na cidade de Iporá, Goiás, para subsidio da tomada de decisão para futuras instalações de geração de energia eólica descentralizadas para o bombeamento de água.

\section{Materiale Métodos}

Para o referido estudo, os dados de velocidade do vento foram obtidos por meio do Posto Meteorológico do INMET (Instituto Nacional de Meteorologia), instalado na fazenda Escola do Instituto Federal Goiano, localizado na cidade de Iporá, Goiás, Brasil $\left(16^{\circ} 26^{\prime}\right.$ $31^{\prime \prime S} ; 51^{\circ} 07^{\prime} 04^{\prime \prime W}$, altitude de $584 \mathrm{~m}$ ), Figura 1.

Figura 1. Localização da cidade de Iporá na região oeste do estado de Goiás (detalhe em preto).

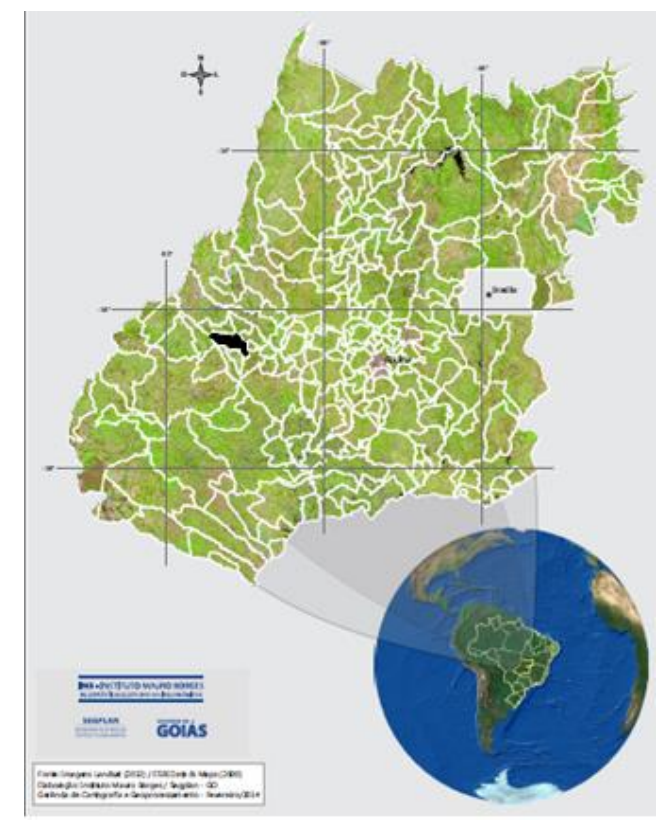

Fonte: Landsat (2013) / ESRI Data \&Maps (2000), Instituto Mauro Borges, Secretaria de Gestão e Planejamento do Estado de Goiás

A velocidade do vento instantânea foi medida com o anemômetro Wind Set WA25 da empresa Vaisala®, instalado em uma torre a dez metros de altura do solo. Para a realização das análises foram utilizados registros de velocidade do vento, entre os anos de 2014/2015.

Para a descrição estatística do regime de ventos, foram organizados os valores diários em ordem decrescente e divididos em intervalos de classes a cada $1 \mathrm{~m} . \mathrm{s}^{-1}$, sendo o intervalo mínimo de 0-1 $\mathrm{m} \mathrm{s}^{-1}$ e o máximo de 8-9 $\mathrm{m} \mathrm{s}^{-1}$. Como era inviável instalar um anemômetro em 
todas as alturas de leitura desejadas, utilizou-se uma fórmula empírica (Equação 1) para simular as velocidades do vento para diferentes alturas $(15 ; 20 ; 25 ; 30 ; 40$ e $50 \mathrm{~m})$, a partir de uma altura padrão de leitura $(10 \mathrm{~m})$. A fórmula empírica utilizada segue a Lei logarítmica de perfil do vento, uma simplificação prática apresentada por Farret (2010).

$$
\frac{u_{1}}{u_{2}}=\frac{\ln \left(\frac{h_{1}}{h_{0}}\right)}{\ln \left(\frac{h_{2}}{h_{0}}\right)}
$$

em que,

$\mathrm{u}_{1}$ - velocidade do vento para a altura $\mathrm{h}_{1}$ de $10 \mathrm{~m}$;

$\mathrm{u}_{2}$ - velocidade do vento para a altura $\mathrm{h}_{2}$ de $15 ; 20 ; 25 ; 30 ; 40$ e $50 \mathrm{~m}$, a parir da superfície do solo;

$\mathrm{h}_{0}$ - comprimento característico da rugosidade do solo $\mathrm{h}_{0}$.

Os valores de $\mathrm{h}_{0}$ foram definidos de acordo com Hassan e Sykes (1990), onde se adotou o valor mínimo para a classificação "relva baixa/estepes”, ou seja, $h_{0}$ igual a 0,01 .

\section{Cálculo dos parâmetros da distribuição de Weibull}

Normalmente, a utilização gráfica de dados de velocidade do vento de séries temporais, obtidas a partir do instrumento de medição, é tão ondulada e irregular que inviabiliza qualquer conclusão a partir desses gráficos. É por isso que uma função de distribuição estatística é muitas vezes utilizada na análise de dados medidos de velocidade do vento (AKOREDE et al., 2013). As duas funções de distribuição mais populares que são utilizadas para o ajuste da distribuição de probabilidade da velocidade do vento, em um dado local em um dado período de tempo, são as distribuições de Rayleigh e Weibull (HUANG E WAN, 2012). Contudo, a distribuição de Rayleigh se torna inapropriada para velocidades do vento abaixo de $4,5 \mathrm{~ms}^{-1}$, assim, deve-se utilizar uma distribuição mais geral como a de Weibull, com a qual é obtida uma melhor aproximação para dados diários e com séries curtas de velocidade do vento(AKOREDE et al., 2013). A função de distribuição de Weibull usada neste estudo é dada na Equação 2, enquanto a função de probabilidade acumulativa correspondente é dada na Equação 3. 


$$
\begin{gathered}
f(u)=\left(\frac{k}{c}\right)\left(\frac{k}{c}\right)^{k-1} \exp \left[-\left(\frac{u}{c}\right)^{k}\right] \\
F(u)=\int_{0}^{u} f(u) d u
\end{gathered}
$$

Substituindo a Equação 2 na Equação 3, obtém-se a Equação 4

$$
f(u)=\int_{0}^{u}\left(\frac{k}{c}\right)\left(\frac{k}{c}\right)^{k-1} \exp \left[-\left(\frac{u}{c}\right)^{k}\right] d u=1-\exp \left[-\left(\frac{u}{c}\right)^{k}\right]
$$

$k$ e $c$ são os parâmetros de ajuste. $k$ é o parâmetro de forma adimensional; $c\left(\mathrm{~m} \mathrm{~s}^{-1}\right)$ é o parâmetro de escala. Para o cálculo dos parâmetros de escala e deforma, foi utilizado o método por regressão linear que faz uso do caráter linear da distribuição acumulada de Weibull, quando aplicada uma transformação logarítmica, conforme metodologia utilizada por Touré (2005). É um método gráfico onde os parâmetros de ajuste foram determinados como segue: a partir da Equação 5.

$$
\ln \{-\ln [1-F(u)]\}=k \ln u-k \ln c
$$

onde $Y$ e $X$ são definidos como se encontra na Equação 6.

$$
Y=\ln \{-\ln [1-\mathrm{F}(\mathrm{u})]\} \quad \mathrm{X}=\ln \mathrm{u}
$$

a Equação 7 pode ser escrita como

$$
Y=k X-k \ln c
$$

Plotando diferentes valores de $Y$ versus $X$, uma linha reta com inclinação $a=\mathrm{k}$ e que intercepta $b=-k \ln$ c é obtida. Contudo, $c$ é dado pela Equação 8.

$$
c=\exp \left(\frac{-b}{a}\right)
$$


Tendo determinado $k$, é necessário calcular o valor da função Gama (CELIK, 2004) para que esta possa ser usada na equação de distribuição densidade de probabilidade. Para caracterizar o local de acordo com o regime eólico, relacionou-se a função Gama com a velocidade média anual do vento, a variância e a densidade de potência média. Os detalhamentos destas relações podem ser encontrados em Grah et al. (2014).Para o cálculo da densidade de potência média, utilizou-se a densidade do ar igual a $1,12 \mathrm{~kg} \mathrm{~m}^{-3}$, considerando uma altitude de $610 \mathrm{~m}$ e uma temperatura média anual de $24,1^{\circ} \mathrm{C}$.

\section{Resultados e Discussão}

Os dados totalizaram 7992 horas de registros coletados, estabelecidos em classes de $0-1 ; 1-2 ; 2-3 ; 3-4 ; 4-5 ; 5-6 ; 7-8$ e 8-9 $\mathrm{m} \mathrm{s}^{-1}$. Foi feita a caracterização da direção do vento por meio da rosa dos ventos, utilizando o software WRPLOT VIEW, da empresa Likes Environmental (Figura 2).

Figura 2. Rosa dos ventos com frequência diária nos anos 2014/2015, demonstrando a direção do vento de maior predominância na cidade de Iporá.

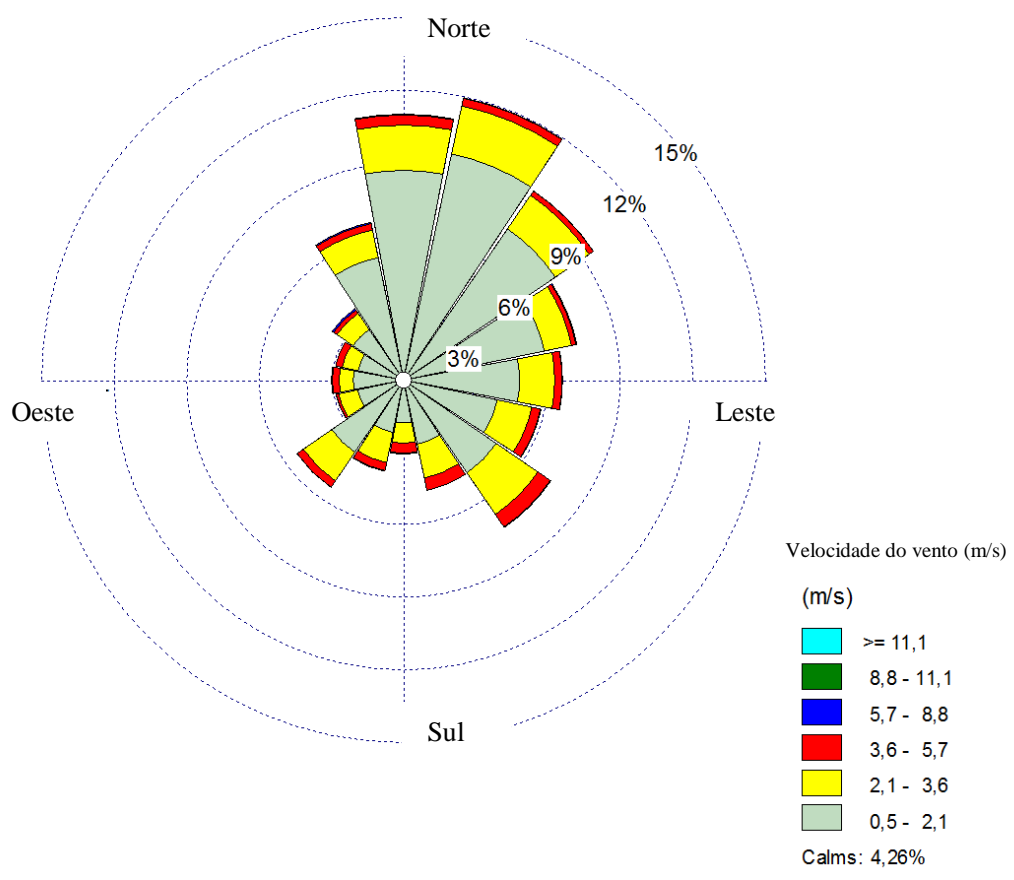

No tocante a referida figura, pode-se destacar a heterogeneidade na direção do vento no local monitorado. Não obstante, é possível identificar a direção norte-sul, com leve tendência de declinação para a direção nordeste-sudoeste, como sendo a direção predominante 
de ventos. A distribuição da velocidade do vento não será um tema de aprofundamento neste estudo, pois, não é um fator utilizado ao se selecionar a viabilidade de um local, esta informação, contudo, é importante para otimização do planejamento do parque eólico (AKOREDE et al., 2013). As turbinas eólicas devem estar posicionadas na direção predominante do vento para um maior aproveitamento energético.

Parâmetros estatísticos de velocidades de vento para o local de estudo são apresentados por dados horários de velocidade a uma altura de $10 \mathrm{~m}$ (Figura 3). A análise deste histograma revela claramente que, os dados de velocidade do vento na faixa de $0-1 \mathrm{~m} \mathrm{~s}^{-}$ 1 , correspondem a $36,95 \%$ dos dados coletados. Totalizando, de 0 até $2,5 \mathrm{~m} \mathrm{~s}^{-1}$, cerca de $72 \%$ do dados de velocidade avaliados. Alavi et al. (2016), em estudo realizado em 5 cidades localizadas na região do leste e sudeste Iraniano, a fim de se caracterizar o padrão de velocidade do vento, apresentam valores predominantes para a faixa de $0-1 \mathrm{~m} \mathrm{~s}^{-1}$ de cerca de $5 \%$, mais especificamente para a localidade de Chabarah. Ainda, mais de $45 \%$ dos valores monitorados de velocidade do vento, apresentam-se próximos de $5 \mathrm{~m} \mathrm{~s}^{-1}$.

Figura 3. Frequência relativa de ocorrência da velocidade do vento na altura de $10 \mathrm{~m}$, a partir da superfície do solo.

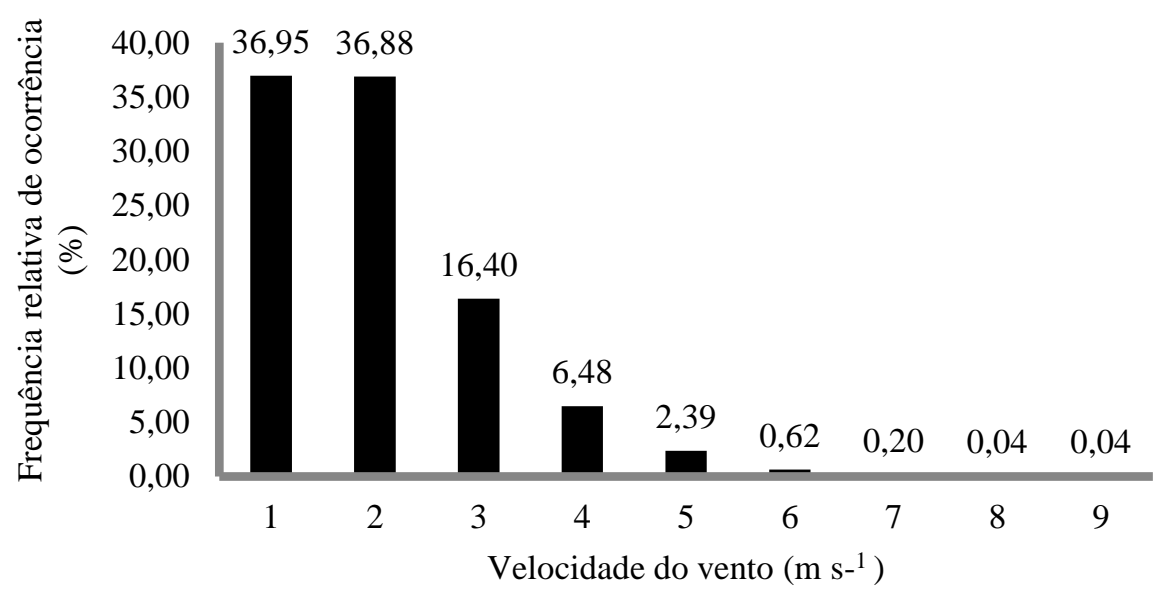

Por outro lado, Carrasco-Díaz et al. (2015), em amplo estudo de diagnóstico do potencial energético realizado na costa do México observaram velocidades do vento, a 10 metros de altura, ocorrendo em $50 \%$ do tempo monitorado, entre valores de 0 a 3,5 $\mathrm{m} \mathrm{s}^{-1}$.

Nesse aspecto, a localidade de Iporá, Goiás, apresenta uma grande disponibilidade de baixas velocidades do vento, fato que, preliminarmente, não é interessante para o investimento em geração de energia eólica. Isso porque a maioria das turbinas eólicas de 
pequena escala não produzem energia útil até a velocidade do vento alcançar 2,5 a 3,0 $\mathrm{m} \mathrm{s}^{-1}$ (AKOREDE, et al., 2013). Este ponto de início comumente é chamado de velocidade de partida, ou ainda, "cut-in".

$\mathrm{Na}$ Figura 4, é possível comparar as velocidades dos ventos para diferentes alturas de instalação da turbina eólica de pequeno porte, onde é possível notar um incremento positivo da velocidade do vento para aumento da altura do eixo da turbina, em relação ao nível do solo. Segundo Safari e Gasore (2010) para projetos envolvendo sistema de conversão eólica, faz-se necessário estimar a velocidade do vento em várias elevações. Segundo, Firtin et al. (2011) as velocidades do vento próximas ao nível do solo são influenciadas, significativamente, por características topográficas do local epelos fatores meteorológicos da área estudada.

Figura 4. Frequência relativa de ocorrência do perfil de velocidade do vento, para as alturas de $10 ; 15 ; 20 ; 25 ; 30 ; 40$ e 50m, a partir da superfície do solo.

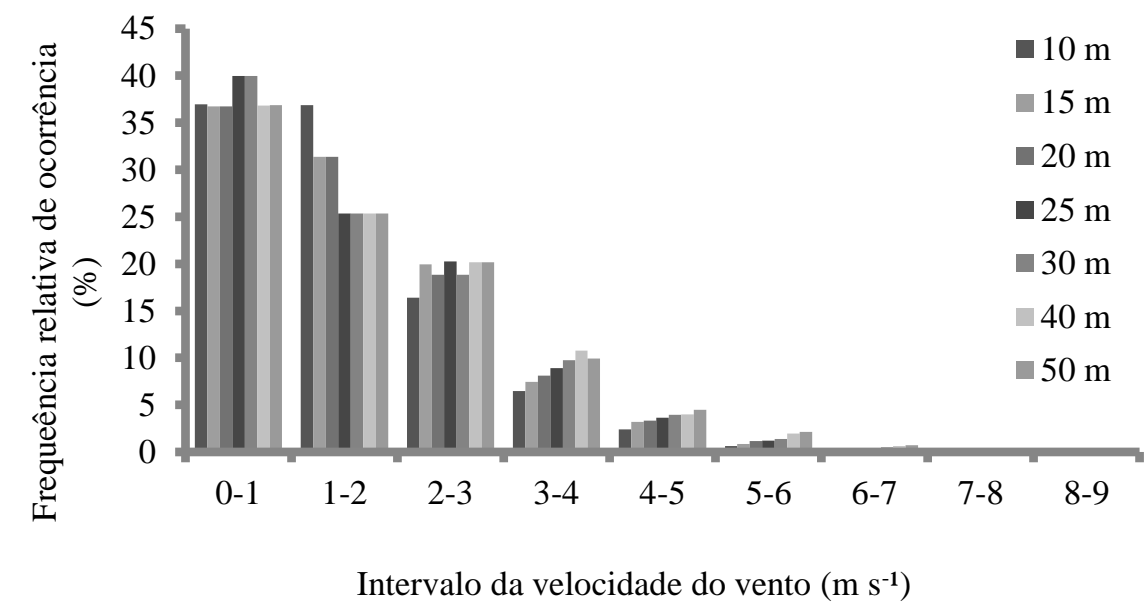

Como, na altura do eixo da turbina de $10 \mathrm{~m}$, a velocidade média medida foi de 1,532 $\mathrm{m} \mathrm{s}^{-1}$, Tabela 1, extrapolou-se a velocidade para outras alturas, contudo, os valores não foram suficientemente superiores. Silva \& Silva (2016), em pesquisa preliminar realizada em Vilhena-RO, divisa entre as regiões Norte e Centro-oeste do Brasil, apresentaram valores médios da velocidade do vento, para a mesma altura, de $3,5 \mathrm{~m} \mathrm{~s}^{-1}$ (ocorrendo em $45 \%$ do tempo). Portanto é preeminente o destaque da necessidade de estudos locais de viabilidade de geração de energia eólica, notadamente devido a discrepância da velocidade média, bem como da frequência da distribuição da velocidade dos ventos entre ambos locais. 
Segundo Fera et al. (2014), apenas em locais que têm na velocidade do vento média anual superior a $6 \mathrm{~m} \mathrm{~s}^{-1}$, devem ser considerados para a energia eólica. Farivarpour et al. (2015), em estudo realizado no noroeste do Iran, na cidade de Tabriz, terceira maior do país, encontraram valores médios de velocidade do vento entre 2,90 e $4,57 \mathrm{~m} \mathrm{~s}^{-1}$.

Tabela 1. Parâmetros estatísticos da velocidade do vento,para as alturas de $10 ; 15 ; 20 ; 25 ; 30$; 40 e $50 \mathrm{~m}$, a partir da superfície do solo.

\begin{tabular}{lccccccc}
\hline & \multicolumn{7}{c}{ Alturas $(\mathrm{m})$} \\
\cline { 2 - 8 } & 10 & 15 & 20 & 25 & 30 & 40 & 50 \\
\hline Média & 1,532 & 1,604 & 1,628 & 1,631 & 1,649 & 1,743 & 1,761 \\
Desviopadrão & 0,932 & 0,982 & 1,022 & 1,092 & 1,113 & 1,146 & 1,181 \\
Variância & 0,869 & 0,963 & 1,045 & 1,193 & 1,239 & 1,314 & 1,396 \\
\hline
\end{tabular}

De acordo com Weekes e Tomlin (2013), a distribuição de probabilidade é definida por uma velocidade média do vento $(c)$ e um fator de forma $(k)$, que descreve a dispersão das velocidades de vento em torno da média. Devido à relação não linear entre a velocidade do vento e a potência eólica, o valor de $k$ terá um impacto sobre a predição na potência do vento. $\mathrm{Na}$ Tabela 2, encontram-se os valores calculados dos parâmetros $k$ e $c$ pelo método da regressão para todas as alturas.

Tabela 2. Valores dos dois parâmetros da distribuição de Weibull para as alturas de 10,15, 20, 25, 30, 40 e 50, a partir da superfície do solo.

\begin{tabular}{cccccccc}
\hline & \multicolumn{7}{c}{ Alturas $(\mathrm{m})$} \\
\cline { 2 - 8 } & 10 & 15 & 20 & 25 & 30 & 40 & 50 \\
\hline$k$ & 1,343 & 1,340 & 1,323 & 1,280 & 1,274 & 1,292 & 1,278 \\
$c$ & 1,669 & 1,747 & 1,768 & 1,760 & 1,778 & 1,885 & 1,900 \\
\hline
\end{tabular}

Analisando os valores de $k$ e $c$ encontrados para a cidade de Iporá, percebe-se que para o parâmetro de forma $(k)$, os valores ficaram próximos de 1 para todas as alturas demonstrando que os ventos de baixa velocidade são predominantes, o que corrobora dados apresentados na Tabela 1, pela velocidade média do vento.Valores de " $k$ " e "c" similares ao deste estudo foram apresentados também por Carrasco-Díaz et al. (2015), para a localidade de Soto La Marina e Altamira, costa leste do México.

$\mathrm{O}$ fator de forma mostra a assimetria da curva de Weibull, altos valores para $k$ (entre 2 e 3) significam que a curva é mais assimétrica para as altas velocidades do vento, e baixos 
valores de $k$ (entre 1 e 2 ) traduzem-se em um formato da curva da referida distribuição como sendo mais assimétrica para baixas velocidade do vento, indicando que estas são mais prováveis (MOSTAFAEIPOUR, 2011). Analisando os valores do parâmetro de escala (c), que também está relacionado com o vento disponível da região, observa-se que os valores são baixos e próximos de 1 o que significa que o valor modal de cada distribuição é o mais representativo do todo, ou seja, mais confiável será empregá-lo como, substituto da média dos valores. O parâmetro de escala $c$ mostra o valor de pico na curva de Weibull. Por outro lado, altos valores de $c$ significam que a dispersão da distribuição ocorre em uma faixa mais larga de velocidades do vento (MOSTAFAEIPOUR, 2011).

A Distribuição de Probabilidade Anual formada a partir dos dados da série temporal de Iporá, Goiás, para todas as alturas calculadas são apresentadas na Figura 5.

Figura 5. Distribuições de probabilidade de velocidade anual dos ventos, calculadas a partir da função de Weibull, para as alturas de 10;15;20;25; 30; 40 e 50 m, a partir da superfície do solo.

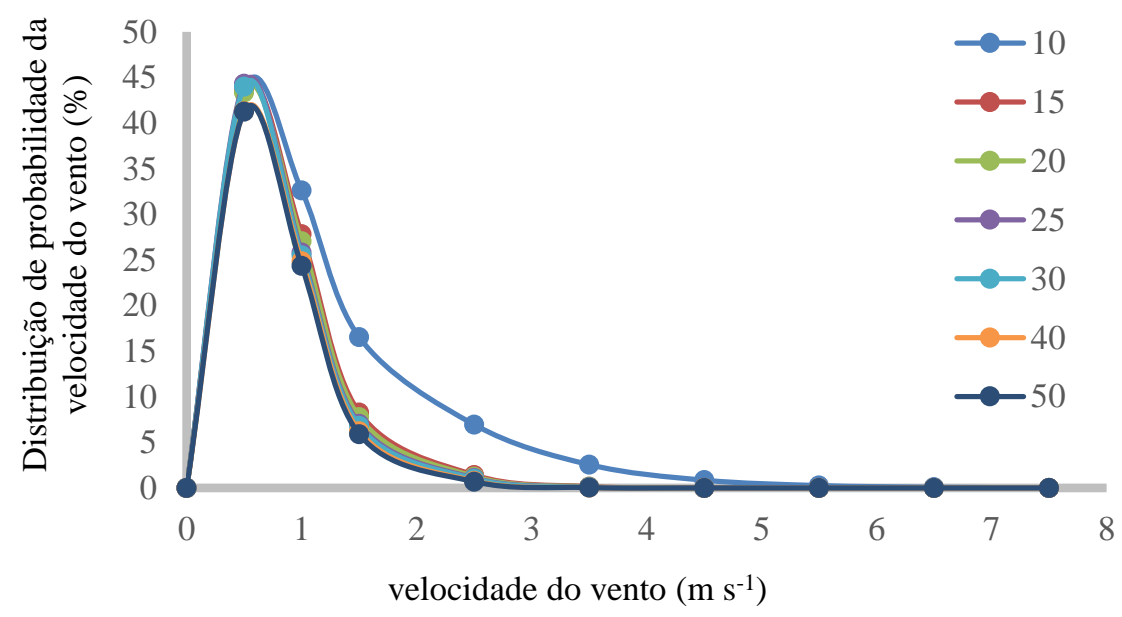

A distribuição da velocidade do vento predominantemente determina o desempenho dos sistemas de energia eólica. Uma vez que a distribuição de velocidade do vento é conhecida, o potencial de energia eólica e, portanto, a viabilidade econômica pode ser facilmente obtida (ZHOU et al., 2006, RAIMUNDO \& SANTOS, 2015). Observa-se nas curvas obtidas e apresentadas na Figura 5 que a velocidade do vento está bem abaixo do indicado para a geração de energia eólica, onde os dados da velocidade do vento para todas as alturas analisadas se concentram próximos a $1 \mathrm{~ms}^{-1}$ e com maior probabilidade de ocorrer ao longo do ano. Segundo Akorede et al. (2013), a velocidade mínima para a geração eólica é 
altamente influenciada pela velocidade de partida das turbinas eólicas comerciais, que está em torno de 2,5 a $3,0 \mathrm{~m} \mathrm{~s}^{-1}$.

A densidade da potência do vento é geralmente considerada um melhor indicador do recurso do vento do que a velocidade do vento (AL-NASARetal., 200 5). Os valores calculados de densidade de potência do vento podem ser visualizados na Figura 6.

Figura 6. Valores calculados de densidade de potência, medido para $10 \mathrm{~m}$ e simulados para as para as alturas de $10 ; 15 ; 20 ; 25 ; 30 ; 40$ e $50 \mathrm{~m}$, a partir da superfície do solo.

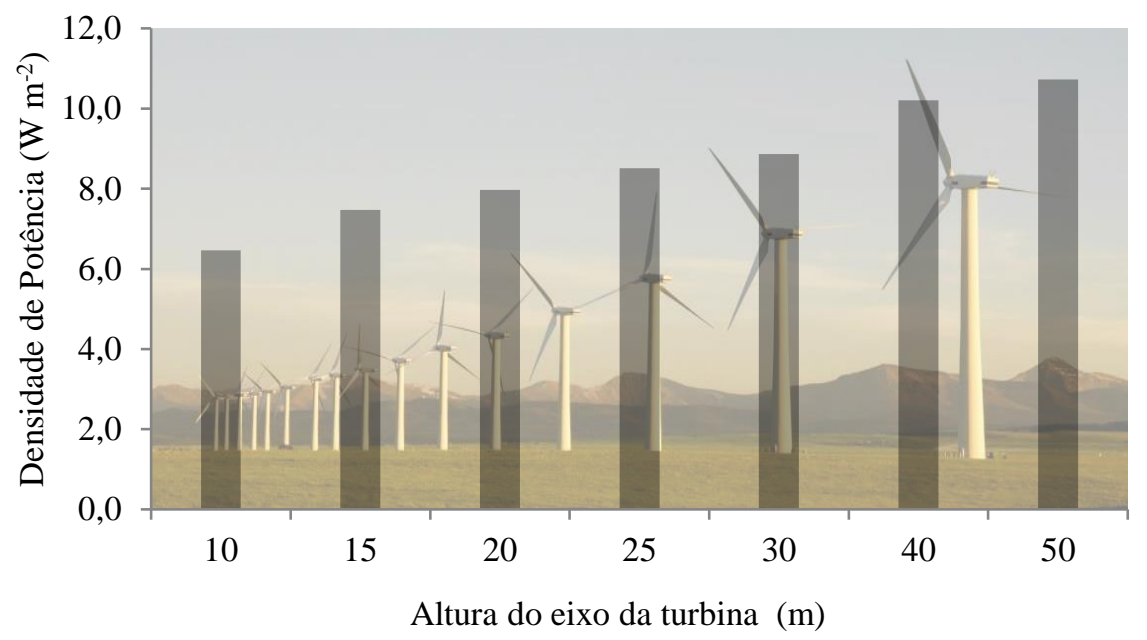

Pela análise da Figura 6, em consonância com classificação apresentada por Mirhosseini et al., (2011), a densidades de potência do vento na cidade de Iporá, para todas as alturas, são classificadas como baixas, com valores entre 6,5 a $10,7 \mathrm{~W} \mathrm{~m}^{-2}$. Segundo os autores, os locais que apresentam densidade de potência menores que $100 \mathrm{~W} \mathrm{~m}^{-2}$ estão na classe de potência 1 e não são apropriados para o aproveitamento de geração de energia eólica. Em outra classificação, a Agência Europeia de Meio Ambiente (2009), European Environment Agency- EEA, afirma que as turbinas eólicas só podem operar efetivamente em velocidades do vento acima de $4,0 \mathrm{~m} \mathrm{~s}^{-1}$, isto significa que o local de estudo não se enquadra em uma classificação aceitável para geração de energia eólica. Ao se comparar os valores calculados de densidade de potência à $30 \mathrm{~m}$, com a classificação de velocidade média do vento proposta por Al-Nasaret al. (2005), observa-se que uma densidade de potência menor que $100 \mathrm{Wm}^{-2}$ é enquadrada como um vento fraco para a geração de energia.

Resultado similar de insuficiência na geração de potência energética a partir de geradores eólicos foram apresentados por Carrasco-Díaz et al. (2015), em 2 pontos da costa leste do México. Os referidos autores destacam ainda que há a possibilidade de haver uma 
mudança da distribuição de frequência da velocidade do vento quando se utiliza dados instantâneos ao invés de dados médios. No presente estudo, empregou-se dados médios horários, o que permite posterior análise com dados instantâneos, quando disponíveis.

Foi observado que o ganho de densidade de potência para a altura de $50 \mathrm{~m}$ foi de 40,2\% em relação à altura de $10 \mathrm{~m}$, esta porcentagem de densidade de potência, contudo, não é suficiente para cobrir o investimento com um TEE, dado o nível tecnológico atual.

Assim, dada a realidade de disponibilidade de dados, bem como o nível tecnológico presente na literatura, Iporá apresenta baixa aptidão para aplicações eólicas conectadas a rede, mas pode ser adequada para situações não conectadas a rede de energia elétrica, como para carregamento de baterias e bombeamento de água (CELIK, 2004).

\section{Conclusão}

Devido ao excelente ajuste linear obtido no processo numérico para o cálculo dos parâmetros de escala e de forma, pode-se afirmar que o modelo de Weibull foi adequado as distribuições de velocidade de vento obtidas, apresentando pequenos erros residuais para a altura de medição e as simuladas. Pela sistematização dos métodos utilizados, com a integração trapezoidal e a regressão linear, para determinar os valores de k e c, pôde-se caracterizar o regime eólico de Iporá.

No contexto de futuras aplicações agrícolas, tais como, alimentação energética para bombeamento de água em projetos de irrigação ou abastecimento humano no meio rural a densidade de potência não se apresentou suficiente para manter o sistema com autonomia energética. Não obstante, a variação do potencial de geração em regiões próximas as desse estudo indicam que futuros esforços devem ser direcionados para investigações locais de produção eólica, o que pode ser parcialmente realizado com o aumento espacial de pontos de monitoramento da velocidade do vento. 


\section{Referências Bibliográficas}

AGÊNCIA NACIONAL DE ENERGIA ELÉTRICA. Capacidade de Geração do Brasil. Disponível

$<$ http://www2.aneel.gov.br/aplicacoes/capacidadebrasil/capacidadebrasil.cfm). Acesso em: 08 de agosto de 2016.

AKOREDE, M.F.; RASHID, M.I.M; SULAIMAN, M.H.; MOHAMED, N.B.; AB GHANI, S.B. Appraising the viability of wind energy conversion systemin the Peninsular Malaysia. Energy Conversionand Management, n.76, p. 801 - 810, 2013

ALBIERO, D.; DAHER, S.; MONTEIRO, L.A.; CANAFÍSTULA, F.J.F. Turbina eólica para agricultura familiar do semiárido com inovações tecnológicas para baixas velocidades de vento. Revista Ciência Agronômica, v. 45, n. 1, p. 186-196, 2014.

ALAVI, O.; MOHAMMADI, K.; MOSTAFAEIPOUR, ALI. Evaluation the suitability of Wind speed probability distribution models: A case of study of east and southeast parts of Iran. Energy Conversionand Management, v. 119, p. 101-108, 2016.

AL-NASSAR, W.; ALHAJRAF, S.; AL-ENIZI, A.; AL-AWADHI, L. Potential wind power generation in the State of Kuwait.Renewable Energy.v. 30, n. 14, p. 2149-2161, 2005.

AMARANTE, O.A.C.;BROWER, M.; ZACK, J.; SÁ, A.L. Atlas do Potencial Eólico Brasileiro. Brasília, p.44, 2001.

BRASIL, Portal Brasil. Em 2014, BNDES investiu R\$ 6,6 bilhões em energia eólica. Disponível em: <http://www.brasil.gov.br/economia-e-emprego/2015/01/em-2014-bndesinvestiu-r-6-6-bilhoes-em-energia-eolica >. Acessoem: 11 de julho de 2016.

CARRASCO-DÍAS, M.; RIVAS, D.; OROZCO-CONTRERAS, M.; SÁNCHEZMONTANTE, O. Na assessment of wind power potential along the coast of Tamaulipas, northeastern Mexico. Renewable Energy. v. 78,p. 295-305, 2015.

CELIK, A.N. A statistical analysis of wind power density based on the Weibull and Rayleigh models at the southern region of Turkey.Renewable Energy.v. 29, n. 4, p. 593-604, 2004.

ERTÜRK, M.The evaluation of feed-in tariff regulation of Turkey for onshore wind energy based on the economic analysis.Energy Policy, v. 45, p.359-367, 2012

EUROPEAN ENVIRONMENT AGENCY.Europe's onshore and offshore wind energy potential: An assessment of environmental and economic constraints. EEA Technical report: Copenhagen, 85 p., 2009

FARIVARPOUR, F.; SOLTANI, S.; SOLTANI, N.; ROSEN, M. A. Assessment of Wind energy potential and economics in the north-western Iranian cities of Tabriz and Asdabil.RenewableandSustainable Energy Reviews, v. 46, p. 87-99, 2015.

FARRET, A.F.Aproveitamento de pequenas fontes de energia elétrica. $2^{\mathrm{a}}$ Ed. Santa Maria: Ed. da UFSM, 2010. 
FERA, M.; IANNONE, R.; MACCHIAROLI, R.; MIRANDA, S.; SCHIRALDI, M.M.; Project appraisal for small and medium size wind energy installation: The Italian wind energy policy effects. Energy Policy, v.74, p.621-631, 2014.

FIRTIN E, GULER O, AKDAG SA. Investigation of wind shear coefficients and theireffect on electrical energy generation.Applied Energy, v.88, p.4097-4105, 2011.

GRAH, V.F.; PONCIANO, I.M.; BOTREL, T.A. Potential for wind energy generation in Piracicaba, SP, Brazil.RevistaBrasileira de EngenhariaAgrícola.v.18, n. 5, p. 559-564, 2014.

GRIESER, B.; SUNAK, Y.; MADLENER, R. Economics of small wind turbines in urban settings: An empirical investigation for Germany. Renewable Energy, v.78, p. 334-350, 2015 .

HASSAN, U.; SYKES, D.M. Wind structure and statistics. In: FRERIS, L.L. (Ed.). Wind energyconversion systems. New York: Prentice Hall, p. 11-32, 1990.

HUANG S.J, WAN H.H. Determination of suitability between wind turbine generators and sites including power density and capacity factor considerations.IEEE TransSustain Energy, v. 3, p.390 - 397, 2012.

IICA- Instituto Interamericano de Cooperação para a Agricultura. Análise Territorial para o Desenvolvimento da Agricultura Irrigada.FEALQ, USP, Piracicaba, 2014.

MIRHOSSEINI, M.; SHARIFI, F.; SEDAGHAT, A. Assessing the wind energy potential locations in province of Semnan in Iran.Renewable and Sustainable Energy Reviews.v.15, n. 1, p. 449-459, 2011.

MOSTAFAEIPOUR, A.; SEDAGHAT, A.; DEHGHAN-NIRI, A.A.; KALANTAR, V. Wind energy feasibility study for city of Shahrbabak in Iran. Renewable and Sustainable Energy Reviews. v. 15, n. 6, p. 2545- 2556, 2011.

RAIMUNDO, D. R.; SANTOS, I. F. S. Estudo de um projeto para geração de energia eólica no Brasil: viabilidade econômica e emissões evitadas. Revista Brasileira de Energias Renováveis. v. 4, p. 65-75, 2015.

SAFARI, B.; GASORE, J.A statistical investigation of wind characteristics and wind energy potential based on the Weibull and Rayleigh models in Rwanda. Renewable Energy. v. 35, n. 12, p. 2874-2880, 2010.

SILVA, R. L.; SILVA, D. L.; Avaliação preliminar do aproveitamento de energia eólica no município de Vilhena-RO. Revista Brasileira de Energias Renováveis. v. 5, n. 4, p. 466$480,2016$.

TOURÉ, S. Investigations on the eigen-coordinates method for the 2-parameter Weibull distribution of wind speed.Renewable Energy. v. 30. p. 511-521, 2005. 
WEEKES, S.M.; TOMLIN, A.S. Evaluation of a semi-empirical model for predicting the wind energy resource relevant to small-scale wind turbines.Renewable Energy, v. 50, n. 2, p. 280-288, 2013.

ZHOU, W.; YANG, H.; FANG, Z.Wind power potential and characteristic analysis of the Pearl River Delta region, China.Renewable Energy. v. 31, n. 6, may, p. 739-753, 2006. 